\section{COMPRAR Y VENDER: TENDENCIAS DE BÚSQUEDA UTILIZANDO GOOGLE TRENDS EN MÉXICO DURANTE EL COVID-19}

\author{
BUYING AND SELLING: TRENDS OF SEARCHING USING GOOGLE \\ TRENDS TOOL IN MEXICO DURING COVID-19
}

\author{
COMPRE E VENDA: TENDÊNCIAS DE PESQUISA USANDO O GOOGLE \\ TRENDS NO MÉXICO DURANTE A COVID-19
}

\section{Julio César Montiel Flores}

Artículo recibido julio 2021 | Arbitrado agosto 2021 | Aceptado septiembre 2021 | Publicado 13 de diciembre 2021

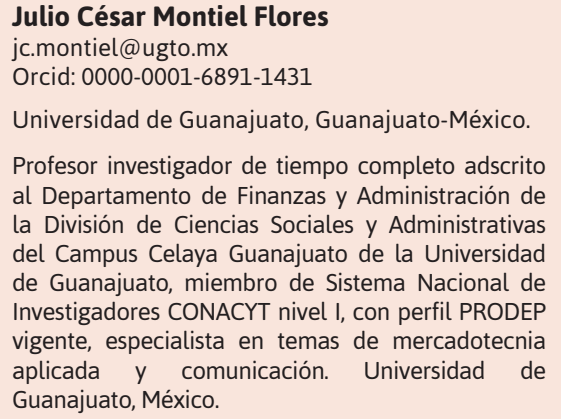

Palabras clave: Tendencias del consumo; Comercio; COVID-19; Google Trends

\begin{abstract}
The purpose of this study is to analyze the information search trends performed by Google search engine users from the period defined from January 1, 2020 to August 26, 2021 using one of Google's functionalities called Google Trends, a free access tool in which it is possible to analyze the popularity of searches performed by people around the world. This tool allows observing the behavior of searches by country and by category defined by Google. Through this analysis it was possible to observe the interest in the purchase and sale of different products and services in Mexico since the beginning of the pandemic.
\end{abstract}

Key words: Consumer trends; Commerce; COVID-19; Google Trends 


\section{Resumo}

O objetivo deste estudo é analisar as tendências de busca de informações realizadas pelos usuários do mecanismo de busca Google de 1 de janeiro de 2020 a 26 de agosto de 2021 utilizando uma das funcionalidades do Google chamada Google Trends, uma ferramenta de acesso livre na qual é possível analisar a popularidade das buscas realizadas por pessoas em todo o mundo. Esta ferramenta permite observar o comportamento das buscas por país e por categoria definida pelo Google. Através desta análise foi possível observar o interesse na compra e venda de diferentes produtos e serviços no México desde o início da pandemia.

Palavras-chave: Tendências do consumidor; Comércio; COVID-19; Google Trends

\section{INTRODUCCIÓN}

Como ya es sabido, el 31 de diciembre de 2019 se informó por parte de la Comisión Municipal de Salud de Wuhan, China de la existencia de casos de neumonía causada por un nuevo coronavirus (Organización Mundial de la Salud, 2021); sin embargo, fue hasta el 11 de marzo de 2020 que la Organización Mundial de la Salud después de varias pruebas y observaciones sobre los niveles de propagación de la enfermedad y su complejidad destacó que el COVID-19 puede alcanzar el nivel de pandemia.

El primer caso de COVID-19 en México se identificó el 27 de febrero de 2020, se trató de un caso de importación de un hombre que viajó a Italia anteriormente; además en el estado de Sinaloa se dio parte de otra persona que se trasladó a ese mismo país (Secretaría de Salud, 2020). Las disposiciones sanitarias establecidas posteriormente estuvieron enmarcadas dentro de la Jornada Nacional de Sana Distancia que dio comienzo a partir del 23 de marzo y hasta el 19 de abril de 2020; en esta jornada se dio prioridad a la ampliación del espacio social entre personas, a las medidas de higiene personal, a la suspensión temporal de las actividades no esenciales, al confinamiento en casa y a la no asistencia a eventos masivos así como al cuidado de adultos mayores, entre otras más (Instituto Mexicano del Seguro Social, 2020).

Sin duda alguna, el impacto económico debido al COVID-19 fue devastador y esto debido al cierre de empresas cuya actividad no fue considerada como esencial, es decir, que toda actividad no relacionada con la rama médica, de seguridad y protección ciudadana, funcionamiento fundamental de la economía, operación social del gobierno, mantenimiento de la infraestructura crítica debía ser suspendida de manera temporal.

Por su parte, Esquivel (2020) afirma que la pandemia también provocó estragos en el gasto de las personas; esto lo explica primeramente por el lado de la oferta, ya que si existió una intención de compra de los consumidores, los negocios se encontraron cerrados por disposiciones oficiales; por el lado, de la demanda se comprende que la menor actividad económica representó pérdida o disminución de ingresos y por ende, el decremento en el consumo de diversos tipos de bienes (p. 4).

Los sectores que sufrieron mayores consecuencias negativas debido a la pandemia fueron y siguen siendo: El turismo, el transporte y el consumo en restaurantes o servicios de comida rápida afirma Esquivel (2020), al 
menos de lo documentado hasta julio de 2020. El turismo dejó de recibir gente en los distintos destinos, los restauranteros tuvieron que idear nuevas estrategias de producción y comercialización para permanecer a flote, la industria manufacturera tuvo que tomar otros rumbos en la producción después de una espera llena de incertidumbre, entre otras situaciones más.

Otro de los aspectos, menciona Esquivel (2020) que marcaron el impacto negativo y la reducción del gasto y del consumo en México fue la pérdida de empleos durante la pandemia, ya que muchas empresas no pudieron sostener la nómina de los empleados al no haber ingresos y terminaron por cerrar.

Esta condición del empleo comenzó a ejercer presión para la recuperación de la normalidad pues como lo afirma Hernández y $\operatorname{Mar}(2020)$ :

\begin{abstract}
"En todo el mundo hay una necesidad por parte de los hogares de ir a trabajar para subsistir, lo que no permite que las medidas de contención de las pandemias sean aplicadas eficazmente. Por lo tanto, los esfuerzos generados para proteger a la población por los gobiernos, se complican, dando como resultado, tensión social en aquellos países que cuentan con una significativa economía informal" (p. 12).
\end{abstract}

La situación como se puede observar desde la perspectiva económica abrió un panorama muy desalentador y lleno de incertidumbre debido a tres expectativas: La elaboración de una vacuna, la reducción de contagios y la recuperación económica paulatina como consecuencia de los dos anteriores.

Por otro lado, a través de sus boletines de prensa el Consejo Nacional de Evaluación de la Política del Desarrollo Social (CONEVAL) informó sobre la condición de la pobreza en México en el período de 2018 y 2020 haciendo hincapié en su incremento pasando de 7 a un 8.6 por ciento, es decir de 8.7 a 10.8 millones de personas en el país respectivamente; en adición, afirma también que la pandemia tuvo impactos negativos en el ingreso, la salud, la educación y la alimentación (CONEVAL, 2021).

Pese a lo anterior, y como consecuencia del confinamiento en el hogar, las personas tuvieron qué reformular las actividades cotidianas, tanto escolares, laborales, de comercio, de relaciones sociales y de entretenimiento; esto llevó a procurar un equilibrio en todo el caos causado por la pandemia en casa y las Tecnologías de la Información y Comunicación (TICS) fueron y siguen siendo herramientas fundamentales para llevar a cabo dichas actividades, entre otras.

Internet se ha convertido en un servicio indispensable en la vida moderna y cotidiana pues esta red de redes de alcance mundial ha refrendado sus beneficios constantemente y más aún en esta pandemia COVID-19 donde los usuarios en todo el mundo han constatado sus bondades para permanecer en un estado de socialización, vinculación y trabajo a la distancia; así también los gobiernos procuraron el apoyo operativo a través de la utilización de las TICS y otros medios para reincorporar actividades productivas (Salazar, 2021). 
La gran cantidad de información que está almacenada en distintos dispositivos interconectados en Internet, la facilidad de conexión, los diversos servicios de información que se han desarrollado para su funcionamiento en la red son bondades significativas que han permitido que las personas y las organizaciones a nivel mundial puedan realizar cientos de funciones a través de sus dispositivos acortando distancias y desarrollar nuevas formas de cohabitar entornos cada vez más complejos.

Sin embargo, la pandemia logró penetrar a los rincones más personales de los círculos sociales, los impactos en las diversas actividades económicas poco a poco fueron afectadas por las disposiciones anticontagio y las prohibiciones de socialización en espacios concurridos en donde el virus podría ocasionar expandirse de manera exorbitante.

Internet fue el recurso que permitió la socialización virtual para cumplir con las actividades presenciales requeridas por esos acuerdos con organizaciones de diversa índole; la educación por ejemplo, sentó los precedentes de un cambio drástico en las prácticas de enseñanza aprendizaje, los sistemas educativos con baja preparación en el uso de las Tecnologías de la Información y Comunicación (TICS) tuvieron que adaptarse a una disruptiva estrategia para atender a distancia los requerimientos de los estudiantes de cualquier grado.

Pese a todas estas circunstancias de crisis parecería que el planeta tomaría una actitud de alerta no solo en cuestiones de salud sino en términos de ahorro, economización y optimización de los recursos de consumo diario y que el gasto durante la pandemia debiera de estructurarse para poder dar prioridad a emergencias debido a diversas situaciones que ya se han mencionado.

La pregunta de investigación que se prevé contestar en esta comunicación es: ¿Cuáles fueron los intereses o intenciones del usuario del buscador Google en términos de compra y venta en un contexto de pandemia en el periodo comprendido entre el primero de enero de 2020 y hasta el 26 de agosto de 2021?

Por lo anterior, el objetivo de esta comunicación es examinar las tendencias sobre el interés en temas relativos a la compra y venta de determinados productos y servicios a partir de Google TRENDS desde el primer día de enero de 2020 hasta el 26 de agosto de 2021.

Los motores de búsqueda son instrumentos indispensables hoy día para encontrar información precisa sobre un tema de interés en particular y que a través de algoritmos precisos logran arrojar resultados relacionados con la palabra o conjuntos de palabras clave en un tiempo muy reducido y por orden de relevancia.

Sin embargo, siguiendo a Pérez-Montoro y Codina (en Injante y Mauricio, 2020, p. 1) “los motores de búsqueda son los sistemas de información documental más importantes de nuestro tiempo", añaden que son una forma que permite explotar la vasta información que existe en la web y que incorporan nuevas funcionalidades día a día a fin de atender las necesidades de información de sus usuarios. 
En el mercado existe varios motores de búsqueda (Mangles en Injante y Mauricio, 2020) pero quien sigue marcando la pauta de uso en el mundo ha sido Google: Un proyecto creado en el año de 1997 en la Universidad de Standford que logró destacarse de su competencia por varias características entre las que destacan la gratuidad, la eficiencia y rapidez de búsqueda, organización y visualización de los resultados que arroja.

Google como corporativo mantiene como misión que consiste en "[...] organizar la información del mundo y hacer que sea útil y accesible para todos" (Google, 2021b, p. 1), está razón de ser empresarial le ha permitido crear diversos productos y servicios de búsqueda y exploración, visualización y reproducción de contenido, dispositivos conocidos como Made by Google, habla y envío de mensajes de texto, organización de contenido, trabajo inteligente, negocios, etcétera.

Uno de los servicios que Google ofrece es Google TRENDS en donde es posible consultar lo que los usuarios en el mundo o bien en una región particular están buscando en un tiempo determinado mediante palabras clave o temas (Google, 2021a). En su página de soporte de Google TRENDS se afirma que lo que arroja esta herramienta es el interés de búsqueda por determinados temas y además advierte que se considera información concreta obtenida de otra más amplia (Google, 2021a).

\section{MÉTODO}

Se trata de un estudio descriptivo que utilizó el servicio de análisis de las búsquedas
Google denominado: Google TRENDS, se delimita la búsqueda espacial en el país México. Además, se habilitaron los límites temporales de búsqueda a través del intervalo de tiempo personalizado de la herramienta entre el 1 de enero de 2020 y 26 de agosto de 2021.

Como bien lo explica el proveedor de la herramienta, Google (2021a) los resultados que arroja no provienen de una encuesta, sino que a partir de la gestión de miles de millones de búsquedas diarias solo se utiliza una muestra de esas búsquedas y los números resultantes se muestran en una escala del 1 al 100 respecto a la proporción de un tema en relación con el total de búsqueda de otros temas.

Otras consideraciones que se distinguen de la herramienta de búsqueda consisten (Google, 2021a) en la utilización de tablas para indicar en una escala del 1 al 100 la frecuencia de búsqueda de los temas más populares lo que significa que un tema evaluado con 100 tiene la máxima popularidad, mientras que un tema con 50 tendrá la mitad de la frecuencia del tema más buscado; adicionalmente se indica que hay temas cuya búsqueda ha aumentado drásticamente a través de la palabra AUMENTO que representa que la frecuencia de búsqueda ha rebasado el $5000 \%$ o bien también se encuentra expresiones en porcentaje explícito para indicar dicho aumento.

Los términos de búsqueda en Google Trends fueron: Comprar y Vender, esto se realizó de manera combinada, es decir, se utilizaron estos dos términos en un solo momento. 
RESULTADOS

Como puede advertirse en la Figura 1, la tendencia comparada en las palabras de búsqueda COMPRAR y VENDER representa un interés significativo por la acción de comprar en contraste con la acción de vender. Adicionalmente la gráfica indica que el interés por comprar parecía ser constante desde la semana 1 hasta la 11 que correspondió desde el 1 de enero de 2020 hasta el 8 de marzo del mismo año; se observa un descenso en las siguientes dos semanas, sin embargo parece crecer el interés por comprar hasta la semana 30 que comienza a partir de 19 de julio de 2020. El punto máximo del interés de comprar se manifestó la semana 52 que coincide con el inicio de la época decembrina después de dos picos altos de las semanas iniciadas el 4 de octubre y 8 de noviembre de 2020 respectivamente.
En el inicio del año 2021 el interés por comprar, según las búsquedas en esta herramienta decrecieron encontrando un pico creciente en la semana que comenzó el 21 de marzo con el inicio de la primavera.

Con respecto al interés de vender, las búsquedas de los usuarios se muestran también en la Figura 1 en donde se observa un comportamiento más regular que en el caso anterior; los picos altos que se aprecian fueron los marcados en las semanas de la 26 a la 33 que correspondió del 21 de junio de 2020 al 15 de agosto de 2020. Para el año de 2021, los picos importantes de mayor interés por vender fueron en la semana iniciada el 4 de abril, 9 de mayo y 18 de julio de 2021 coincidentemente con el inicio de la Semana Santa, el día de la madre y el período vacacional de verano en México.

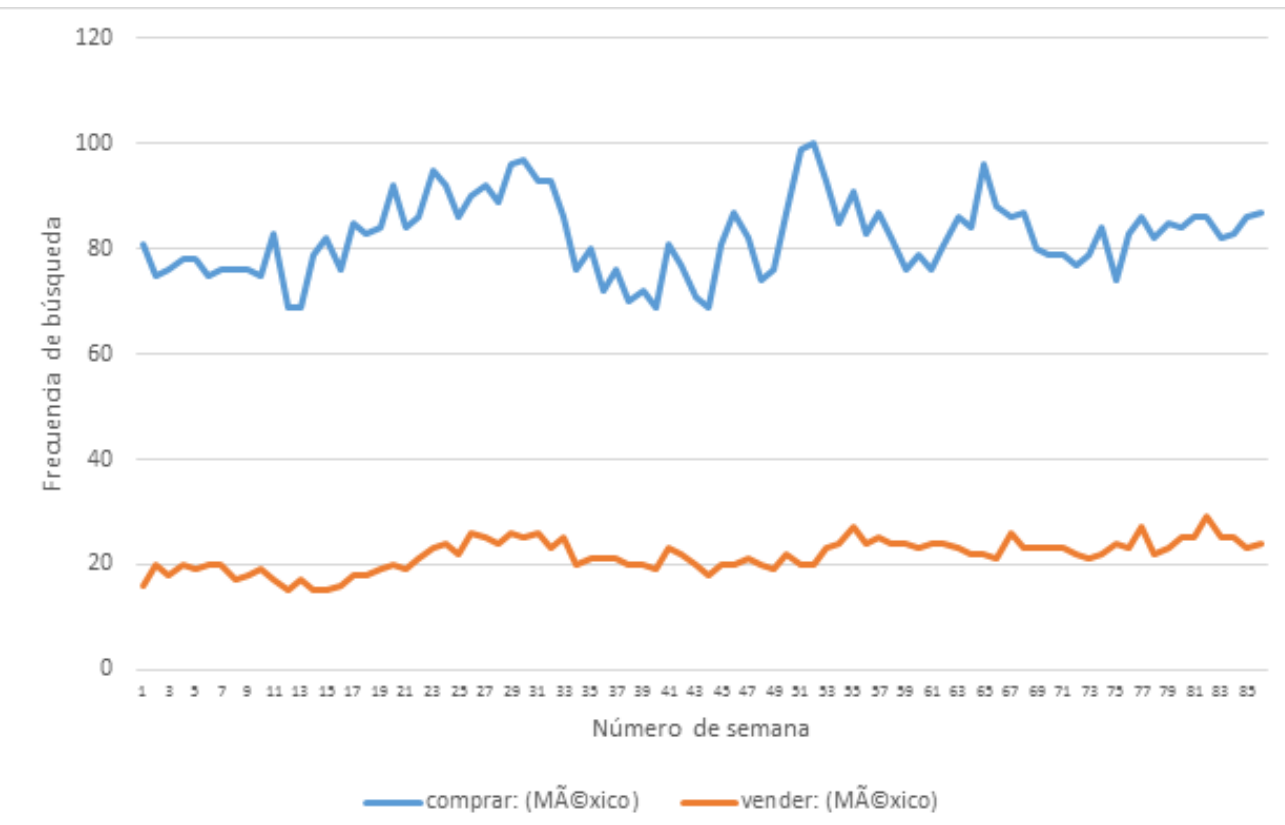

Figura 1. Tendencia comparada de las búsquedas en Google Trends entre COMPRAR y VENDER. Fuente: Elaboración propia con base a los datos arrojados por Google TRENDS

Nota. La frecuencia de búsqueda se expresa en una escala del 1 al 100 a partir de la muestra utilizada por Google Trends. 
En la Tabla 1 se exhiben las consultas más frecuentes relacionadas con el interés de comprar, es importante recordar que Google Trends utiliza una escala del 1 al 100 para indicar la frecuencia de consulta entre la que destaca para este caso el concepto de LUGAR principalmente como dos de las consultas más frecuentes. Se destaca también la forma de compra, en particular EN LíNEA, así como tiendas virtuales particulares; con menor frecuencia se hacen visibles los productos posibles de comprar sobre los que se muestra el interés como lo son: acciones, autos, saldo, dólares y criptomonedas.

Tabla 1. Principales consultas con relación a la palabra COMPRAR por los usuarios de Google en el período referido y su popularidad.

\begin{tabular}{lc}
\hline \multicolumn{1}{c}{ Consultas más frecuentes } & Popularidad (Escala 1-100) \\
\hline Dónde comprar & 100 \\
Dónde & 98 \\
comprar en línea & 6 \\
comprar en amazon & 3 \\
comprar acciones & 3 \\
comprar auto & 3 \\
comprar saldo & 1 \\
comprar dólares & 1 \\
comprar bitcoin & 1 \\
cómo comprar acciones & 1 \\
\hline
\end{tabular}

Por su parte, en la Tabla 2 se da muestra del comportamiento de las búsquedas relacionadas con el interés de comprar, se destaca un aumento significativo con la compra de saludos y criptomonedas tipo dogecoin, las cuáles fueron creadas desde el 2013 y han tenido mucha aceptación por el apoyo de figuras públicas en el mundo. Estas búsquedas relacionadas aumentaron de forma importante.
En menor escala se encuentran las búsquedas de tiendas virtuales particulares como Amazon, Price Shoes, Coppel, Alibabá y Telcel. Así mismo, las búsquedas de productos singulares como perros, tarjetas Sodexo para el apoyo empresarial a sus empleados o Robux, monedas virtuales relacionadas con videojuegos son también muy visibles según la información arrojada. 
Tabla 2. Consultas relacionadas a las principales por los usuarios de Google en el período referido y su estado de incremento de búsqueda.

\section{Búsquedas relacionadas}

comprar saludos

dónde comprar dogecoin

dogecoin precio

comprar dogecoin

Dogecoin

comprar saludos de famosos

cómo comprar robux

comprar acciones de amazon

me voy a comprar un perro

comprar en línea price shoes

comprar en línea coppel

cómo comprar acciones

comprar bitcoin

comprar acciones

tarjeta sodexo

cómo comprar en alibaba

comprar internet telcel

sodexo donde comprar

se puede comprar terreno con infonavit

cómo comprar bitcoin

cómo comprar acciones de amazon

\section{Estado del incremento}

Aumento

Aumento

Aumento

$+4,000 \%$

$+4,000 \%$

$+3,100 \%$

$+500 \%$

$+400 \%$

$+350 \%$

$+250 \%$

$+250 \%$

$+250 \%$

$+200 \%$

$+200 \%$

$+190 \%$

$+180 \%$

$+170 \%$

$+170 \%$

$+150 \%$

$+140 \%$

$+120 \%$
En la Tabla 3 se concentran las principales consultas con relación al interés por vender, en una escala de popularidad marcada por Google Trends, las búsquedas más populares corresponden a "cómo vender" y en seguida le continúa "qué vender".

Se distingue un interés menor pero visible de "vender en casa" o bien, vender desde otras alternativas virtuales como Mercado Libre y Amazon. Entre los productos que destacan bajo el interés de vender son: monedas, ropa, fotos, comida y frases. 
Tabla 3. Principales consultas con relación a la palabra VENDER por los usuarios de Google en el período referido y su popularidad.

\begin{tabular}{|c|c|}
\hline Consultas más frecuentes & Popularidad (Escala 1-100) \\
\hline cómo vender & 100 \\
\hline qué vender & 75 \\
\hline vender casa & 24 \\
\hline qué es vender & 16 \\
\hline vender en mercado libre & 14 \\
\hline qué puedo vender & 11 \\
\hline dónde vender monedas & 11 \\
\hline ropa para vender & 11 \\
\hline amazon vender & 10 \\
\hline vender fotos & 9 \\
\hline productos para vender & 9 \\
\hline cosas para vender & 8 \\
\hline vender por internet & 8 \\
\hline vender en amazon & 8 \\
\hline como vender en mercado libre & 8 \\
\hline moneda de 20 pesos & 7 \\
\hline postres para vender & 7 \\
\hline vender moneda de 20 pesos & 7 \\
\hline comida para vender & 7 \\
\hline frases para vender & 5 \\
\hline cómo vender ropa & 5 \\
\hline qué se puede vender & 5 \\
\hline ideas para vender & 5 \\
\hline cómo vender en amazon & 5 \\
\hline dónde vender monedas antiguas & 4 \\
\hline
\end{tabular}

Con respecto a las búsquedas relacionadas con el interés de vender, la Tabla 4 concentra las categorías que aumentaron de manera reveladora como lo son los productos con interés de venta como son: Fotos de pies, monedas, billetes, principalmente. Se manifiesta en las búsquedas relacionadas un interés por conocer formas de venta ya sea a través de alguna página en Facebook o la forma en que puede obtenerse una licencia para la venta de alcohol en el Estado de México o incluso generar ideas de productos a vender en fechas específicas del año, en este caso el 14 de febrero en que se festeja el día de San Valentín en México, considerado como el día del amor y la amistad. 
Tabla 4. Consultas relacionadas a las principales por los usuarios de Google en el período referido y su estado de incremento de búsqueda.

\section{Búsquedas relacionadas}

vender fotos de pies

moneda de 20 pesos Veracruz

moneda de 20 pesos Veracruz dónde vender

vender billete de 100 pesos

dónde vender billetes de 100

dónde vender billete de 100 pesos

vender fotos de mis pies

moneda de 20 pesos 500 años de Veracruz dónde vender

cómo vender fotos de mis pies

moneda de 20 pesos Veracruz precio donde vender

dónde vender billetes de 100 pesos

qué vender en cuarentena

dónde puedo vender mi moneda de 20 pesos

dónde puedo vender billetes de 100 pesos

billetes de 100 nuevos

billete de 100 serie AA

dónde vender un billete de 100 pesos

cómo crear una página en facebook para vender

dónde puedo vender mi billete de 100 pesos

qué vender el 14 de febrero

cómo vender billete de 100 pesos

valor de moneda de 20 pesos

cómo vender un billete de 100 pesos

vender en copreterito

permiso para vender alcohol Estado de México 2020

\section{Estado del incremento}

Aumento

Aumento

Aumento

Aumento

Aumento

Aumento

Aumento

Aumento

Aumento

Aumento

Aumento

Aumento

Aumento

Aumento

Aumento

Aumento

Aumento

Aumento

Aumento

Aumento

Aumento

Aumento

Aumento

Aumento

Aumento

\section{Discusión}

En el estudio de venta online que presenta en el 2021 la Asociación Mexicana de Venta Online (AMVO, 2021) se subraya el contexto pandémico ocasionado por el virus SARCOV-2 vivido en México y el comportamiento de compra de los internautas mayores a 18 años; este estudio fue realizado en ocho levantamientos de información en el período del 8 de abril de 2020 hasta el 13 de enero de 2021.

Entre los resultados más relevantes de dicho estudio se enmarcan los sentimientos de los consumidores frente a la pandemia entre los cuales prevalece un sentimiento de aislamiento durante los primeros meses de 
la pandemia, los encuestados de ese estudio comentaron que sus salidas se limitaron a la compra de comida, emergencias médicas y restringieron el contacto con otras personas.

Además, los encuestados tomaron medidas de precaución a la salida al trabajo y limitar cualquier salida; sin embargo al tener que salir el miedo al contagio fue una constante y manifestaron, según dicho estudio, que la pandemia tendría un impacto en temas de dinero ocasionando angustia e incertidumbre por no saber qué iba a pasar en el futuro.

El estudio de la AMVO (2021) se subraya el interés de los encuestados por buscar en internet dónde comprar productos y servicios de manera importante, ya que casi el $50 \%$ de sus encuestados de un total de 1861 personas afirmó haberlo hecho. Desde octubre de 2020 a enero de 2021.

Entre los productos y servicios de mayor interés para comprar en línea durante los primeros meses de la pandemia (AMVO, 2021) por importancia se mencionan: Comida (Alimentos congelados, frutas, verduras, enlatados), medicamentos, tecnología, medios de entretenimiento, así como productos para la salud y bienestar físico, espiritual y psicológico).

Para enero de 2021, dicho informe comenta que los principales productos de interés por comprar de los encuestados son: Comida a domicilio, tecnología, supermercado, medicamentos, medios y entretenimiento $y$ productos para el aseo del hogar.

Sin duda las prioridades en el interés de compra cambiaron en el tiempo mientras la pandemia tomó su curso; el informe no incluye información con respecto al interés por vender.

En contraste, se reconoce en primera instancia, que el sentimiento generado en el consumidor durante pandemia estuvo ensombrecido por un sentido de angustia e impacto negativa en los temas de dinero y que las prioridades sobre el interés por comprar productos y servicios evolucionaron en el tiempo.

Aunque el método del estudio publicado por la AMVO es distinto al de esta comunicación es posible afirmar que:

Los datos comparativos arrojados por la herramienta de Google Trends en el período del 1 de enero de 2020 al 26 de agosto de 2021 muestran un claro y mayor interés en lo relacionado a la palabra COMPRAR en comparación con la búsqueda del opuesto: VENDER.

Al revisar las fechas en calendario y de acuerdo con el número de semana que correspondían los datos de la figura 1 , es necesario decir que, el interés de los usuarios de Google por la acción de compra tuvo importantes picos que marcaron una tendencia al alza comenzando el 12 de abril de 2020 manteniéndose hasta el 1 de agosto de 2020. El incremento sobre el interés por la actividad de compra se reactivó de manera significativa durante el periodo decembrino, comenzando de 20 de diciembre y hasta el 2 de enero de 2021.

Es importante subrayar que las búsquedas más populares se concentraron en un lugar apropiado para adquirir productos o servicios; mayormente popular resultó la búsqueda de compra a través de Amazon que es una 
de empresas con presencia importante en el mundo en la venta de productos en línea.

Los lugares o sitios en línea de mayor referencia en la búsqueda relacionados a la actividad de compra se encuentran: Amazon, Alibaba, Coppel, Price Shoes, Telcel e Infonavit.

Entre los productos más populares en la búsqueda relativa a la compra se encontraron acciones, saludos de famosos, perros, autos, dólares, bitcoin, robux ${ }^{1}$, dogecoin ${ }^{2}$, saldo y tarjetas SODEXO3.

En lo que respecta al interés por la acción de VENDER puede observarse en la Figura 1 que en los meses de junio, julio y agosto del 2020 los usuarios del motor de búsqueda Google estuvieron más interesados por vender, así como los primeros tres meses del 2021. Sin embargo, se mantiene un comportamiento del interés en la acción de vender sin fluctuaciones muy marcadas.

Adicionalmente la acción de VENDER estuvo relacionada con dos cuestionamientos base: Qué vender y cómo vender. Los objetos más populares relacionados como más populares fueron: casa, monedas, ropa, fotos, comida, frases, billetes, permisos para vender alcohol.

1 Robux es una moneda virtual, la cual se puede obtenerse bajo compra en los sitios oficiales de Roblox que corresponde a una plataforma de juegos en línea, los Robux también pueden ganarse a través de los juegos que tiene la misma plataforma. (Roblox, 2021)

2 El dogecoin es un tipo de criptomoneda similar al bitcoin que sirve para realizar transacciones desde redes descentralizadas (Rivera, 2021).

3 Las tarjetas SODEXO son otorgadas por la empresa del mismo nombre para proporcionar servicios de alimentación, uniformes, gasolina y otras compensaciones con el fin de incentivar la productividad de los trabajadores de las empresas en convenio (SODEXO, 2021).
Entre los lugares que reflejaron mayor interés como medio de venta estuvieron: Mercado libre, Amazon y Facebook. Se destaca el interés por vender en una fecha del año como búsqueda más popular relacionada al 14 de febrero que en México se festeja el día del San Valentín o del amor y la amistad.

Llama particularmente la atención el interés manifestado por los usuarios de la búsqueda de Google por vender fotos de pies el cual aumentó considerablemente durante el período.

\section{CONCLUSIONES}

A manera de conclusiones pudo observarse que durante el período conformado desde el primero de enero de 2020 y hasta el 26 de agosto de 2021, el interés de búsqueda de los usuarios del motor de Google estuve centrado mayormente en la acción de comprar que en la de vender y en un sentido muy particular pareciera que la pandemia abrió posibilidades para que las personas en México estuvieran más interesadas en adquirir productos y servicios.

Esto pone en perspectiva la idea de un periodo fatalista donde parecía que la pandemia provocada del COVID-19 alargaría más la posibilidad de la recuperación económica y por ende las familias en México tendrían qué ver posibilidades más radicales de subsistencia debido a los largos períodos primeramente por el confinamiento, segundo por la apertura de negocios y tercero por la idea de una "nueva normalidad" cada vez menos posible. 
Como ya se ha dicho, la herramienta de Google Trends solo arroja los resultados del interés sobre uno o varios términos de búsqueda y que en este caso fue comparada sobre acciones opuestas entre COMPRAR y VENDER. En este sentido se identifica un contraste muy visible entre estas acciones.

Sin duda, el interés sobre la actividad comercial y de intercambio manifestado en Google Trends puede otorgar un reflejo de que la compra está relacionada más con el entretenimiento mientras que su opuesto vender conserva un sentido mayor de obtención de ingreso.

\section{REFERENCIAS}

AMVO (2021). Estudio de Venta Online. Asociación Mexicana de Venta Online. file:///C:/Users/julio/Downloads/ AMVO_Estudio_de_Venta_Online_2021_ Versi\%C3\%B3nP\%C3\%BAblica-3.pdf

CONEVAL (2021). CONEVAL presenta las estimaciones de pobreza multidimensional 2018 y 2020. Comunicado 09. Consejo Nacional de Evaluación de la Política de Desarrollo Social (CONEVAL) https:// www.coneval.org.mx/SalaPrensa/ Comunicadosprensa/Documents/2021/ COMUNICADO_009_MEDICION_ POBREZA_2020.pdf

Esquivel,G.(2020).Losimpactoseconómicosdela pandemia en México. https://www.banxico. org.mx/publicaciones-y-prensa/articulos-yotras-publicaciones/\%7BD442A596-6F43D1B5-6686-64A2CF2F371B\%7D.pdf

Google. (2021a). Preguntas frecuentes sobre los datos de Google Trends. https://support. google.com/trends/answer/4365533?hl=es
Google. (2021b). Página oficial de soporte Google en español. https://support.google. com/websearch/?hl=es\#topic=3378866

Hernández A., y Mar J. (2020). Efectos de la pandemia en la economía Familiar. Revista Economía Actual. 13 (3). Julio-Septiembre 2020. http://economia.uaemex.mx/ Publicaciones/e1303/Efectos\%20de\%20 la\%20pandemia\%20en\%20la\%20economa. pdf

Injante, R., y Mauricio, D. (2020). Método para recomendar factores de posicionamiento personalizados en el motor de búsqueda de Google. Revista Española de Documentación Científica, 43(1), eneromarzo 2020, e253. https://doi.org/https:// doi.org/10.3989/redc.2020.1.1628

Instituto Mexicano del Seguro Social, I. (2020). Para disminuir la propagación del COVID-19, el IMSS promueve las medidas de sana distancia. http://www.imss.gob.mx/ prensa/archivo/202003/142

Organización Mundial de la Salud, O. (2021). Nuevo coronavirus 2019. https://www. who.int/es/news/item/27-04-2020-whotimeline---covid-19https://www.who. int/es/emergencies/diseases/novelcoronavirus-2019?gclid=CjwKCAjwrPCG BhALEiwAUI9X01HEtgxxodSjrbPAuNaR_ y7h49Yu7nKeJobAkzHIfJNiCDgZdj2ojxoC_ hUQAvD_BwE

Rivera, J. A. (2021). Esto es lo que debes saber si quieres comprar un dogecoin. Diario $\mathrm{El}$ Economista. 25 de febrero de 2021. https:// www.eleconomista.com.mx/mercados/ Esto-es-lo-que-debes-saber-si-quierescomprar-dogecoin-20210225-0068.html 
ROBLOX (2021). Sitio Oficial. https://en.help. roblox.com/hc/es/articles/203313200Maneras-de-como-obtener-Robux

Secretaría de Salud, S. (2020). Se confirma en México caso importado de coronavirus COVID- 19. In. https://www.gob.mx/salud/ prensa/077-se-confirma-en-mexico-casoimportado-de-coronavirus-covid-19
Salazar, L. (2021). Innovación, tecnología y MIPYMES en tiempos de la COVID-19. Internet y Pandemia en las Américas. Primera crisis sanitaria en la era digital. Konrad Adenauer Stiftung.

SODEXO (2021). Sitio Oficial. https://www. sodexo.com.mx/ 